\title{
Are the cytoplasmic microstructures within GBM pathologically significant?
}

\author{
Tatsuo Sakai
}

Received: 6 September 2008/Published online: 8 November 2008

(C) Japanese Society of Nephrology 2008

The current issue of CEN features the so-called podocytic infolding glomerulopathy, which is characterized by the emergence of spherical or tubular cytoplasmic microstructures in association with cytoplasmic projections of podocytes within the thickened glomerular basement membrane (GBM) in electron micrographs as well as nonargyrophilic holes in the GBM at light microscopic levels [1]. This type of glomerular change has been recently proposed as a possible new pathological entity in Japan. The three original articles and 15 case reports in this issue are the first survey of the cases with this type of glomerular pathology.

The GBM and other basement membranes are made up by self-assembly of constituent molecules, such as collagen type IV and laminin, which are secreted by the adjacent parenchymal cells. The established basement membranes are thought to be a stable structure, providing a mechanical support and constant milieu for the parenchymal cells. Reflecting the structural and functional stability of basement membranes, cytoplasmic microstructures and cellular projections have been only rarely reported within the basement membranes. One of those rare observations includes the basement membranes of the proximal convoluted tubule, which contains occasional cytoplasmic vesicles at the concavity of the tubular bend [2]. These vesicles are thought to be torn off from the basal cytoplasm of the proximal tubular cells due to higher mechanical stress at this location, and preserved within the basement membranes which shield the vesicles from disposal by

\section{T. Sakai $(\bowtie)$}

Department of Anatomy and Life Structure,

Graduate School of Medicine, Juntendo University,

2-1-1 Hongo, Bunkyo-ku, Tokyo 113-8421, Japan

e-mail: tatsuo@juntendo.ac.jp interstitial cells. In this issue, Fujigaki et al. [3] showed clearly that the microstructures in the GBM are connected with the cellular processes of podocytes by three dimensional analysis.

Another example of heterogeneity of the basement membrane was found around the ductal epithelium of excretory glands, including the liver, pancreas, lacrimal glands and salivary glands $[4,5]$, where the cellular projections of ductal epithelial cells were associated multilaminar basement membranes and fine collagen fibrils. The multilaminar lamina densa together with fine collagen fibrils were thought to be produced by the epithelial cells. The functional significance of cellular projection of ductal epithelial cells are unknown, but may be correlated the extremely high mechanical demand of this epithelium.

The 25 cases reported in this issue revealed clearly that the podocyte infolding glomerulopathy represents not solitary unreproducible observations, but constantly reproducible ones, although the incidence is extremely low. The unique microscopic appearance suggests the common pathological process of these cases, but the etiology is obviously diverse. In this issue, Masuda et al. [6] reported a different type of glomerulopathy with cytoplasmic microstructures within GBM, and suggested that they represented cellular debris trapped during the remodeling of the GBM.

What does the morphology of podocyte infolding glomerulopathy indicate? It would be to some extent rationalized to suppose that the podocytes in this condition are surrendered to more mechanical demand to produce more GBM materials and make basal projections which are torn off to deliver cytoplasmic microstructures to be remained for considerable period in GBM. The paucity of the cases and the diversity of the etiology at present prevent further analysis of the pathology. 


\section{References}

1. Joh K, Taguchi T, Shigematsu H, Kobayashi Y, Sato H, Nishi S, Katafuchi R, Nomura S, Fujigaki Y, Utsunomiya Y, Sugiyama H, Saito T, Makino H. Proposal of podocyte infolding glomerulopathy: A review of 25 cases from nationwide research in Japan. Clin Exp Nephrol. 2008. doi:10.1007/s10157-008-0104-z.

2. Hijikata T, Sakai T. Structural heterogeneity of the basement membrane in the rat proximal tubule. Cell Tissue Res. 1991;266:11-22.

3. Fujigaki Y, Muranaka Y, Sakakima M, Ohta I, Sakao Y, Fujikura T, Sun Y, Katafuchi R, Joh K, Hishida A. Analysis of intra-GBM microstructures in a SLE case with glomerulopathy associated with podocytec infolding. Clin Exp Nephrol. 2008. doi:10.1007/s10157008-0095-9.
4. Hosoyamada Y, Kurihara H, Sakai T. Ultrastructural localization and size distribution of collagen fibrils in Glisson's sheath of rat liver: implications for mechanical environment and possible producing cells. J Anat. 2000;196:327-40.

5. Hosoyamada Y, Sakai T. The ultrastructure of periductal connective tissue and distinctive populations of collagen fibrils associated with ductal ephithelia of exocrine glands. Arch Histol Cytol. 2003;66:407-18.

6. Masuda Y, Mii A, Shimizu A, Fujita E, Aki K, Ishikawa K, Ishizaki M, Sato S, Hayama N, Iino Y, Katayama Y, Fukuda Y. Invagination and infolding of podocytes in glomerular basement membrane in the cases of primary membranous nephropathy. Clin Exp Nephrol. 2008. doi:10.1007/s10157-008-0100-3. 